\title{
El conjunto histórico de Talavera de la Reina (Toledo): Balance y perspectivas
}

\section{Sergio de la Llave Muñoz ${ }^{a}$}

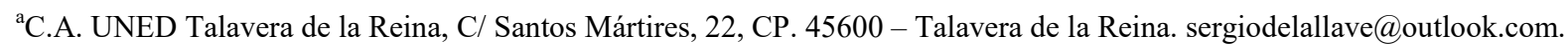

\begin{abstract}
Resumen
Talavera de la Reina ha conservado una ingente cantidad de edificaciones monumentales, a los que se añaden otros inmuebles y espacios de singular interés. Todo ello, llevó en 2016 a varios colectivos a considerar que la ciudad reunía requisitos suficientes para lograr la declaración de Conjunto Histórico. La declaración fue obtenida en febrero de 2019. No obstante, pese a los logros alcanzados, es necesario trazar una estrategia encaminada a crear herramientas y mecanismos destinados a gestionar de forma óptima el Conjunto Histórico. Resulta imprescindible redactar un nuevo Plan Especial destinado a resolver cuestiones como la uniformidad de diferentes elementos del paisaje urbano -mobiliario, cartelerías, sistemas de iluminación, pavimentos...-, establecer criterios sobre el empleo de cerámica artística sobre fachadas, la cesión de alturas a cambio de la conservación de restos arqueológicos, peatonalización de calles o temas de señalización. A ello se suma la necesaria adopción de medidas correctoras sobre fachadas u elementos discordantes con inmuebles o espacios protegidos. Esta serie de acciones requiere la creación de mejoras administrativas o un órgano destinado a centralizar las gestiones relacionadas.
\end{abstract}

Palabras clave: patrimonio cultural, paisaje urbano, Bien de Interés Cultural, gestión del patrimonio.

\begin{abstract}
A great quatity of monuments have been preserved in Talavera de la Reina, apart from other sites and unique spaces. In 2006, this led several social groups to consider that the city met the requirements to get the Historic Centre declaration. This was achieved in February 2019, although it is now necessary to present a strategy that leads to the creation of tools and mechanisms that allow its correct management. It is crucial to elaborate a new urban plan aimed to solve different issues, like the uniformity of several elements in the urban landscape -urban furniture, signs, lighting systems, pavement...-, establishing criteria on the use of artistic ceramic in façades, allowing extra floors in exchange for archaeological remains preservation, pedestrianizing streets or signage issues. There is also the need to take any corrective measures regarding façades or other disturbing elements in protected spaces or buildings. These actions require the improvement of administratives procedures or the creation of an organism that would centralize all related issues.
\end{abstract}

Keywords: cultural heritage, urban landscape, Bien de Interés Cultural, heritage management. 


\section{Introducción}

Talavera de la Reina se encuentra ubicada en el sector noroccidental de Castilla-La Mancha y es uno de los conjuntos históricos más representativos de la región. Su dilatada historia está marcada por toda serie de avatares históricos de diversa relevancia. Desde la Antigüedad, ha desempeñado un importante rol administrativo de carácter comarcal. Su ubicación, en una zona vadeable del rio Tajo sobre el que se construye un puente en época romana, otorgó a la ciudad el papel de nodo de comunicaciones que conserva hasta la actualidad. En época medieval, supuso una estratégica posición para la defensa y vigilancia de su entorno localizado en la Marca Media, de la que es testigo su alcazaba y su recinto amurallado. Posteriormente, se consolidará como la cabeza de un amplio alfoz conocido como las Tierras de Talavera. La tradición alfarera de etapas anteriores se consolidó a partir del siglo XVI, llegando a convertirse en uno de los principales centros de producción cerámica de la corona de Castilla. A partir de mediados del siglo XVIII, comienza el declive de la ciudad con la aparición de la porcelana y la consiguiente disminución de la producción de loza. La situación empeoró notablemente con la Guerra de la Independencia y diferentes avatares propios del siglo XIX. No será hasta la Restauración alfonsina cuando la ciudad comienza a recuperar progresivamente su empuje de tiempos pasados. Los siglos XIX y gran parte del XX serán catastróficos para la conservación de numerosos elementos inmuebles de la ciudad, fenómenos como las desamortizaciones, la dejadez política o la especulación urbanística han permitido que se perdieran monumentos de forma irreversible.

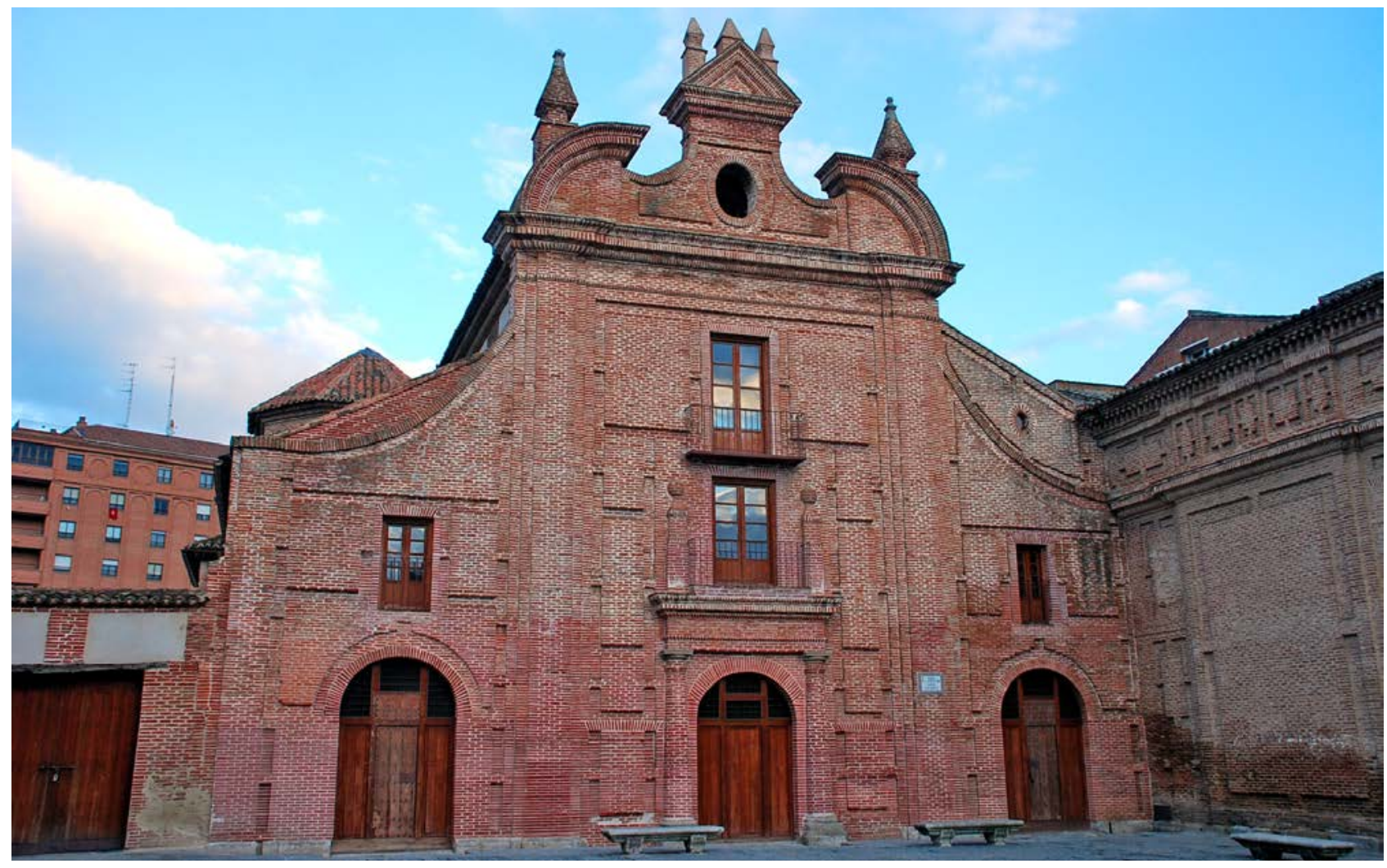

Fig. 1 La Iglesia de San Agustín es uno de los bienes más singulares del Conjunto Histórico de Talavera de la Reina

Pese a los acontecimientos propios de la historia, Talavera de la Reina ha conservado una ingente cantidad de edificaciones monumentales, a los que se añaden otros inmuebles y paisajes urbanos de singular interés, como el entorno de la Plaza de San Agustín (Fig. 1). Todo ello, llevó en 2016 a varios colectivos a considerar que la ciudad reunía requisitos suficientes para lograr la declaración de Conjunto Histórico. Finalmente, tras diversas reuniones y la revisión de la documentación entregada a la Viceconsejería de Cultura de la administración regional, se obtuvo la declaración como Conjunto Histórico en febrero de 2019 (DOCM, 28 de febrero de 2019). No obstante, pese a los logros obtenidos, es necesario trazar una estrategia encaminada a crear herramientas y mecanismos destinados a gestionar el Conjunto Histórico de Talavera de la Reina. 


\section{Génesis y desarrollo}

En la sesión de la comisión del Plan Especial de Protección y Recuperación de la Villa de Talavera de la Reina (PEVT), celebrada el 15 de diciembre de 2015, se hizo la propuesta de declarar, bajo la figura de Conjunto Histórico, el entorno del primer recinto amurallado de la ciudad, la cual fue aprobada por unanimidad. En la sesión del 12 de enero de 2016, se aprobó iniciar los trámites legales de dicha declaración, tanto del primer recinto amurallado como de espacios exteriores con valor histórico-artístico (Alguacil, Maquedano y Molina, 2017).

El 2 de febrero, fue publicado en La Voz del Tajo el artículo de Javier Fernández, "Para que el casco de Talavera se declare Conjunto Histórico", en el que se da cuenta que la iniciativa partía de la Asociación de Vecinos San Jerónimo, contando con el apoyo del Colegio de Arquitectos de Castilla-La Mancha, la Comisión de seguimiento del PEVT, el colectivo cultural La Enrramá y el Colectivo de Investigación Histórica Arrabal. Pocos días después, el 18 de febrero, se sumó a la iniciativa la Fundación Tagus.

El 5 de abril, el diario La Cerca informaba que Santiago Rodríguez-Gimeno, redactor del Plan Especial de la Villa a comienzos de la pasada década de los 90, sería encargado de coordinar el proceso de la declaración. Por decreto, el 9 de junio, se adjudicó al arquitecto Santiago Rodríguez-Gimeno el contrato de servicios de consultoría en la $1^{\text {a }}$ fase de los trabajos (estrategia de actuación, actuaciones previas de identificación de actores y entrevistas correspondientes, elaboración de ponencias preparatorias a jornadas participativas y su evaluación).

En la comisión de seguimiento del PEVT que tuvo lugar el 27 de septiembre, Santiago Rodríguez-Gimeno expuso el contenido del trabajo realizado hasta el momento, explicando las reuniones mantenidas y los pasos a seguir. A los pocos días, el propio Rodríguez-Gimeno participó en una charla-debate sobre "Regeneración de identidad" encuadrada en la IX Semana Internacional de la Arquitectura.

El 2 de noviembre, en un desayuno informativo, Jesús Carrobles, director de la Real Academia de Bellas Artes y ciencias Históricas de Toledo (RABACHT), junto con Rodríguez-Gimeno expusieron el apoyo y el proceso, respectivamente, de la declaración. El día 17, se celebró en el Centro Cultural Rafael Morales una jornada abierta a toda la ciudadanía con el objetivo de dar a conocer y participar en el debate sobre la posible declaración.

Terminada la $1^{\mathrm{a}}$ fase de los trabajos planteados, se adjudicó mediante decreto de 14 de diciembre, a Rodríguez-Gimeno la redacción de un documento orientado a la solicitud de la declaración; es decir, la $2^{\mathrm{a}}$ fase del proceso. Un día después, la RABACHT celebró una Sesión extraordinaria en el Salón de Plenos del ayuntamiento, donde su presidente, Jesús Carrobles, mostró su apoyo expreso a la declaración.

El 10 de enero de 2017, Rodríguez-Gimeno remitió un avance de su trabajo que dio pie a la celebración de varios encuentros con la Dirección Provincial de Cultura, el 18 de enero y el 3 de marzo, donde se abordaron diferentes cuestiones de carácter técnico.

El 1 de febrero, se desarrolló una reunión con el Consejero de Educación, Cultura y Deportes a la que asistieron el alcalde, Jaime Ramos, y la concejala de patrimonio, María Rodríguez, con el fin de encontrar el apoyo necesario para que la declaración fuese un hecho. El 21 de marzo, María Rodríguez, envió al Viceconsejero de Cultura, Jesús Carrascosa, y a la Jefa del Servicio de Patrimonio y Arqueología, María Perlines, copia del documento definitivo redactado por Rodríguez-Gimeno.

El 10 de mayo, tuvo lugar la reunión del Alcalde, Jaime Ramos, acompañado de Rodríguez-Gimeno, María Rodríguez; la responsable de Patrimonio Histórico, Cristina Sánchez; los miembros de la RABACHT, César Pacheco y Miguel Méndez; con el Viceconsejero de Cultura, Jesús Carrascosa y María Perlines, para estudiar el borrador elaborado por Rodríguez-Gimeno y llegar a un consenso sobre el mismo.

Durante el 13 de junio, se desarrolló una reunión entre Rodríguez-Gimeno, técnicos del Ayuntamiento y la Consejería para aboradar diferentes cuestiones sobre la estructura y el contenido del documento.

El 19 de septiembre, se celebró otra reunión en la Consejería de Educación, Cultura y Deporte donde asistieron el Consejero (Ángel Felpeto), el Vicenconsejero de Cultura (Jesús Carrascosa), la Jefa de Servicio de Patrimonio y 
Arqueología (María Perlines), el Jefe de la Sección de Bienes de Interés Cultural (Bienvenido Maquedano), el alcalde de la ciudad (Jaime Ramos), la portavoz municipal (María Rodríguez) y el arquitecto municipal (Gonzalo Bauluz). En la misma, se manifestó que la Viceconsejería no contaba con una solicitud de declaración debidamente registrada. Así pues, con fecha de 6 de octubre fue registrada bajo el título de Solicitud de declaración del Casco Antiguo de Talavera de la Reina como conjunto histórico.

El 25 de septiembre, se realizó una rueda de prensa para anunciar la aprobación, en Pleno Municipal, del documento de trabajo que Rodríguez-Gimeno redactó bajo el título "Talavera de la Reina Conjunto Histórico". Ese mismo día, las comisiones del PEVT y Ordenación y Medio Ambiente dictaminaron, de forma unánime y favorable, solicitar ante la consejería la declaración conforme al documento elaborado. Así pues, el día 28, se aprobó de forma unánime el documento en el Pleno Municipal. Transcurridos unos días, durante la X Semana de la Arquitectura, Rodríguez-Gimeno expuso el documento "Talavera de la Reina Conjunto Histórico" ante los asistentes.

El año 2018 estuvo marcado por la tramitación y revisión documental por parte de la Viceconsejería de Cultura. Es reseñable que, en la XVII edición de los premios San Jerónimo, celebrada por la asociación de vecinos del casco en el mes de abril, Rodríguez-Gimeno fue galardonado por su labor como redactor y coordinador del documento.

La demora en resolver la declaración por parte de la administración regional conllevó, a principios de noviembre, a la solicitud formal del Ayuntamiento de Talavera de la Reina en agilizar los trámites. En este sentido, por resolución de la Viceconsejería de Cultura de 15/11/2018, se inició expediente para la declaración de la Villa de Talavera de la Reina (Toledo) como Bien de Interés Cultural, con la categoría de Conjunto Histórico (DOCM, 20 de noviembre de 2018).

No será hasta el mes de febrero de 2019 cuando finalmente se declare definitivamente por acuerdo de 19/02/2019 del Consejo de Gobierno, por el que se declara Bien de Interés Cultural el Conjunto Histórico de Talavera de la Reina, con la categoría de Conjunto Histórico (DOCM, 28 de febrero de 2019).

Por último, reseñamos el descubriento, a comienzos de octubre, de una placa conmemorativa de cerámica en la que reza la siguiente leyenda: "Talavera de la Reina fue declarada Bien de Interés Cultural con categoría de Conjunto Histórico el 19 de febrero de 2019. La XVIII edición del Premio San Jerónimo al Patrimonio otorga un premio extraordinario a todas las personas y entidades que lo han hecho posible con su unión y trabajo".

\section{Justificación de la declaración}

La declaración de conjunto histórico supone un medio para adquirir una serie de valores que benefician a Talavera de la Reina como garantía de una mejor conservación y puesta en valor de sus bienes culturales y como instrumento hacia la regeneración urbanística de su casco antiguo. En la propuesta inicial de Rodríguez-Gimeno (2018), la zona a declarar comprendía el entorno del primer recinto amurallado, el cual contaba con medidas de protección inscritas en el PEVT, aunque no se descartaba incluir los otros entornos pertenecientes al segundo y tercer recinto amurallado. De igual modo, se planteaba inscribir elementos y espacios culturales ubicados en otros lugares, como los Jardines del Prado, la Plaza de Toros o los Molinos de Abajo.

Por su parte, la delimitación del entorno de protección se fundamentó a partir de la defensa y puesta en valor del ambiente inmediato con el objeto de que las actuaciones que se realicen en inmuebles y espacios contribuyan a mejorar el ambiente en el que se inscribe. De igual modo, se buscaba preservar la percepción visual para evitar un efecto frontera entre el Conjunto Histórico y el resto de la ciudad. Al mismo tiempo, se buscaba la protección de la trama urbana y de los espacios públicos, que son resultado del devenir histórico.

En relación con los espacios del arrabal inmediato (calle Arco de San Pedro, Plaza del Rejoj y Corredera del Cristo), si bien no siempre conservan su edificación antigua en condiciones óptimas, mantienen la trama urbana de referencia. En cuanto al ámbito en torno a la Basílica del Prado y los Jardines del Prado, suponen un refuerzo ambiental y simbólico que ha apoyado siempre la imagen histórica de la ciudad, caracterizada también por ese espacio, antesala multifuncional de la antigua Alameda. 
También se incorporaba el entorno ribereño del Tajo que, formando parte del un paisaje cultural, ha sido pieza inseparable de la lectura del conjunto urbano y debe ser atendido como respaldo paisajístico y ambiental. Sin embargo, la precaria justificación de integración del mismo ha servido para excluirlo del ámbito de declaración.

Finalmente, la delimitación del entorno ha sido justificada adquiriendo gran parte de los planteamientos iniciales de la propuesta. El entorno de protección se extiende hacia el sur debido a la necesidad de mantener la integración del río Tajo con la ciudad y con el fin de proteger sus márgenes y entorno visual. También se incluyen varios inmuebles que configuran la Plaza del Reloj y cuya transformación afecta a los valores ambientales del Conjunto Histórico, así como el colegio Juan Ramón Jiménez, ubicado en la Plaza de El Salvador. El resto del entorno se ciñe a los viales consolidados (Carnicerías, Ronda del Cañillo, Charcón) con el fin de preservarlos de transformaciones futuras. Se consideran incluidas dentro del entorno de protección todas las parcelas excluidas del objeto de la declaración por albergar edificios de nulo valor patrimonial y potente afección visual (Fig. 2). El objetivo que se persigue con su inclusión es el desarrollo de acciones urbanísticas que tiendan a paliar o revertir dichos impactos. De igual modo, se busca la protección de la trama urbana y espacios públicos que, producto del desarrollo urbano desde la edad media, han configurado la actual ciudad.

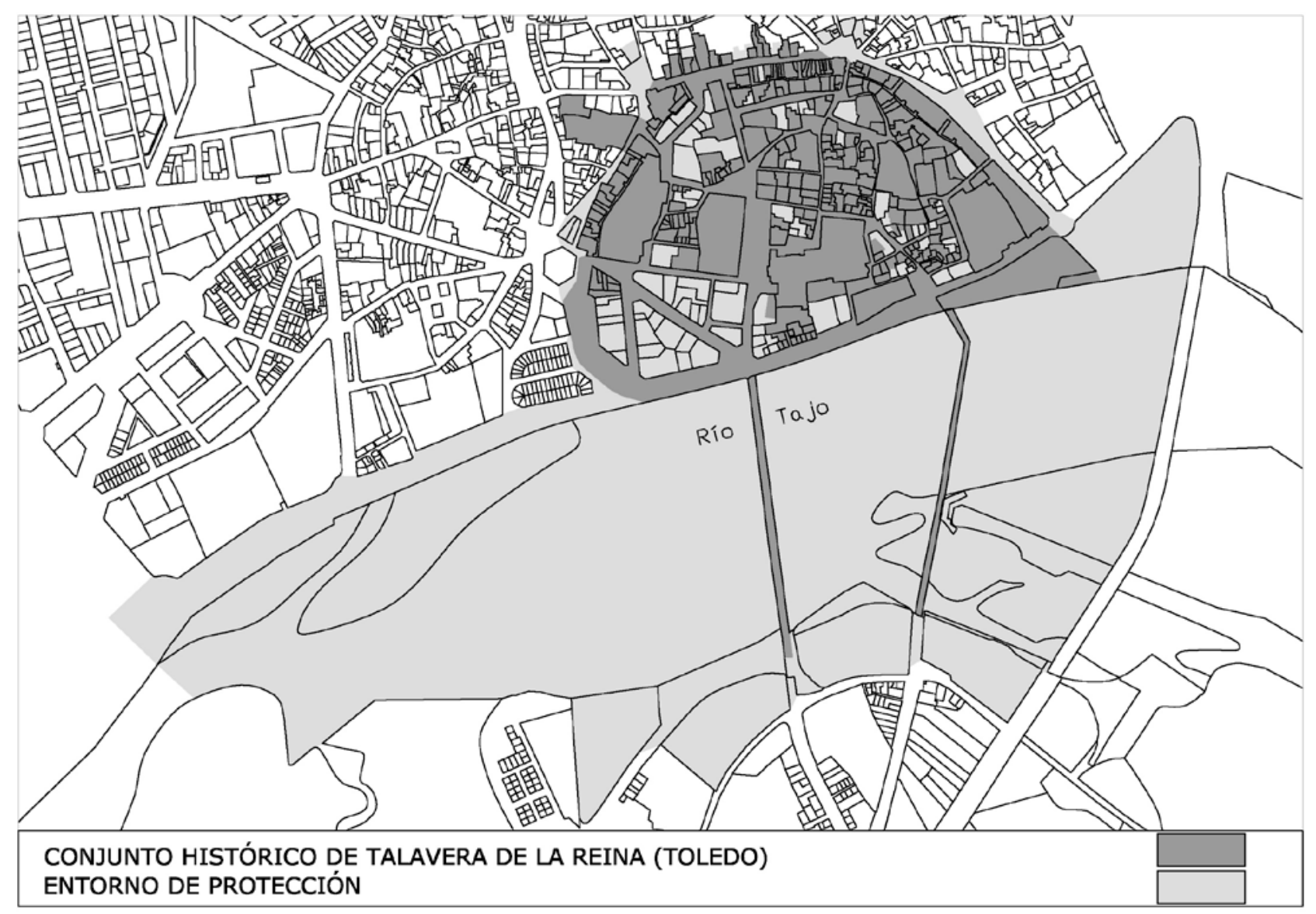

Fuente: DOCM, Año XXXVII, 226, 20 de noviembre de 2018

Fig. 2 Entorno de declaración y protección del Conjunto Histórico de Talavera de la Reina

Una mención aparte merecen los Jardines del Prado. Como se ha visto, el planteamiento original de Rodríguez-Gimeno contemplaba su inclusión dentro del ámbito de declaración de Conjunto Histórico. Sin embargo, la administración regional ha entendido más conveniente declararlo de forma independiente a partir del acuerdo de 19/02/2019, con la categoría de Jardín Histórico (DOCM, 28 de febrero de 2019).

En cuanto a las medidas de protección, están sujetas a lo establecido en el artículo 36 de la Ley 4/2013 sobre Patrimonio Cultural de Castilla-La Mancha (DOCM, 16 de mayo de 2013). De este modo, el Conjunto Histórico de Talavera de la Reina, cuenta con la máxima protección y tutela, y su utilización estará siempre subordinada a que no se ponga en 
peligro su conservación y sus valores. Cualquier cambio de uso, segregación o agregación, habrán de ser autorizados por la Consejería competente en materia de Patrimonio Cultural (DOCM, 28 de febrero de 2019).

\section{Diversidad vs uniformidad del paisaje urbano}

Una vez realizada la declaración de Conjunto Histórico, nos encontramos con deficiencias en relación con la aplicación de criterios homogéneos, acciones de gestión eficaces o la ejecución de medidas correctoras. Es decir, entender el paisaje urbano del Conjunto como algo unitario pese a su diversidad arquitectónica, circunstancia que se da en ámbitos como en el recinto fortificado de la ciudad (Fig. 3).

El actual PEVT, a través de su Comisión de Seguimiento, regula cuestiones urbanísticas como criterios a seguir sobre fachadas (tonalidad, materiales, ventanas, puertas, etc.). Sin embargo, es menester proceder a la adopción de medidas correctoras destinadas a rebajar el impacto visual de numerosos elementos que actualmente conviven en el entorno del Conjunto Histórico. Es el caso de la ubicación de contenedores, aparcamientos, marquesinas, señalización o publicidad. De igual modo, resultaría interesante intensificar la regulación sobre terrazas hosteleras (tonalidad, tipo de mobiliario, publicidad...), la presencia o retirada de elementos vegetales sobre monumentos o espacios inmediatos (arbolado, jardinería) o la existencia de estructuras arquitectónicas y elementos asociados discordantes (escaleras, pérgolas, cableados, cuadros eléctricos o el propio mobiliario urbano).

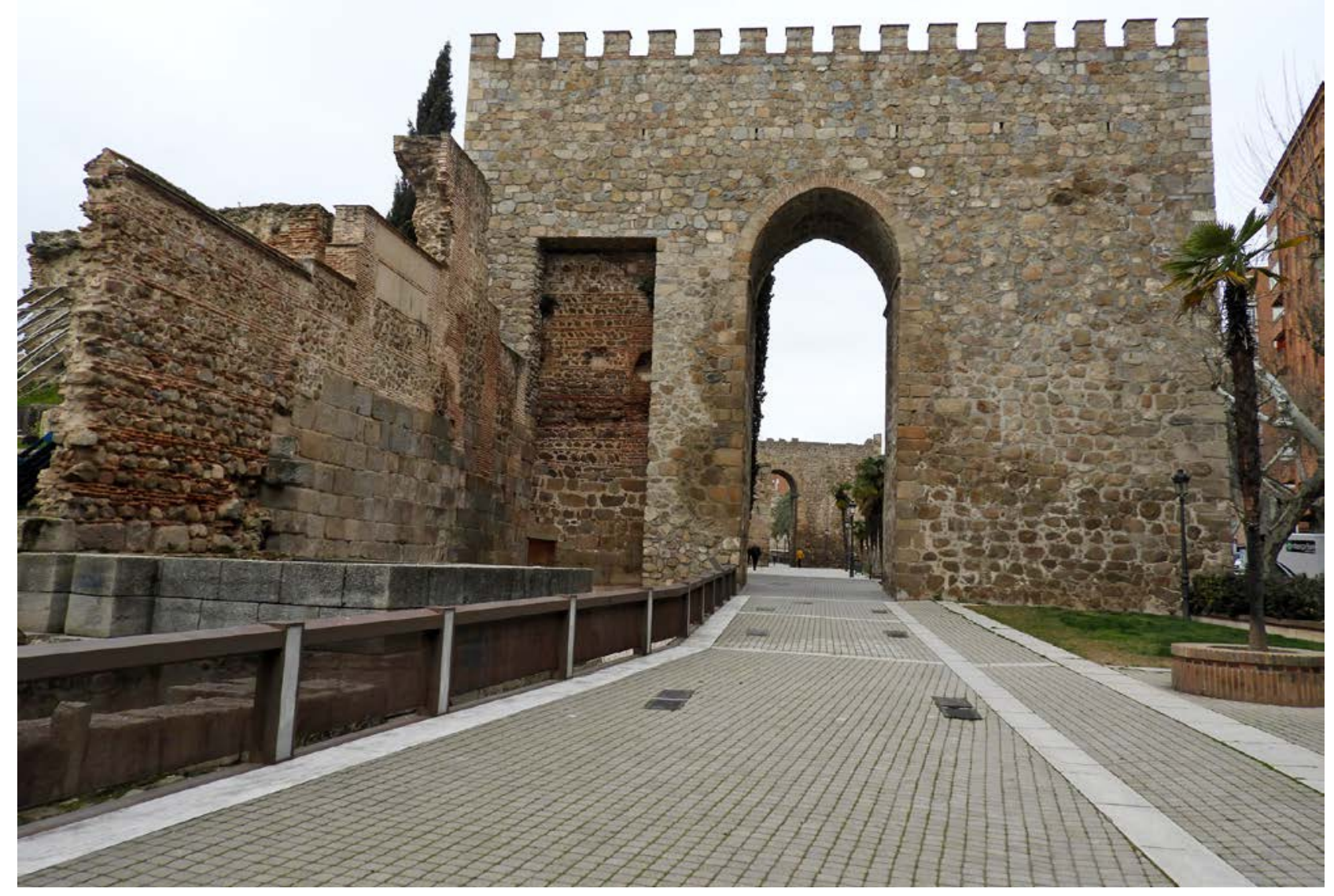

Fuente: De la Llave, 2020

Fig. 3 EI Paisaje urbano del recinto amurallado en la calle Carnicerías

Bajo una serie de planteamientos similares, se precisa la necesidad de buscar criterios comunes sobre elementos que forman parte del paisaje urbano, como pavimentos (más de 15 variedades), bolardos (hasta 9 tipos), bancos (más de 8 clases), farolas (hasta 7 variedades), papeleras (hasta 7 tipos) o vallados (más de 4 clases). En el caso que nos ocupa, las regeneraciones urbanas realizadas a través del programa Urban en la ciudad muestran la ausencia de comunicación entre los proyectos desarrollados entre la calle Charcón, la plaza de El Salvador y la Corredera del Cristo. En citados 
espacios, puede comprobarse la adopción de criterios que marcan verdaderos conflictos visuales entre el patrimonio cultural y diferentes elementos discordantes instalados en su entorno.

\section{Prospectiva de futuro}

Gran parte de las medidas citadas anteriormente requieren ser recogidas y reguladas en un nuevo Plan Especial. Este documento deberá estar directamente coordinado con otros instrumentos de gestión como el Plan de Ordenación Municipal (Ezquiaga, 2008), el futuro Plan Director de Muralla, planes de movilidad y accesibilidad, señalización, regeneración urbana, recogida de residuos domésticos o el Plan de Mantenimiento del Patrimonio Histórico Municipal, entre otros.

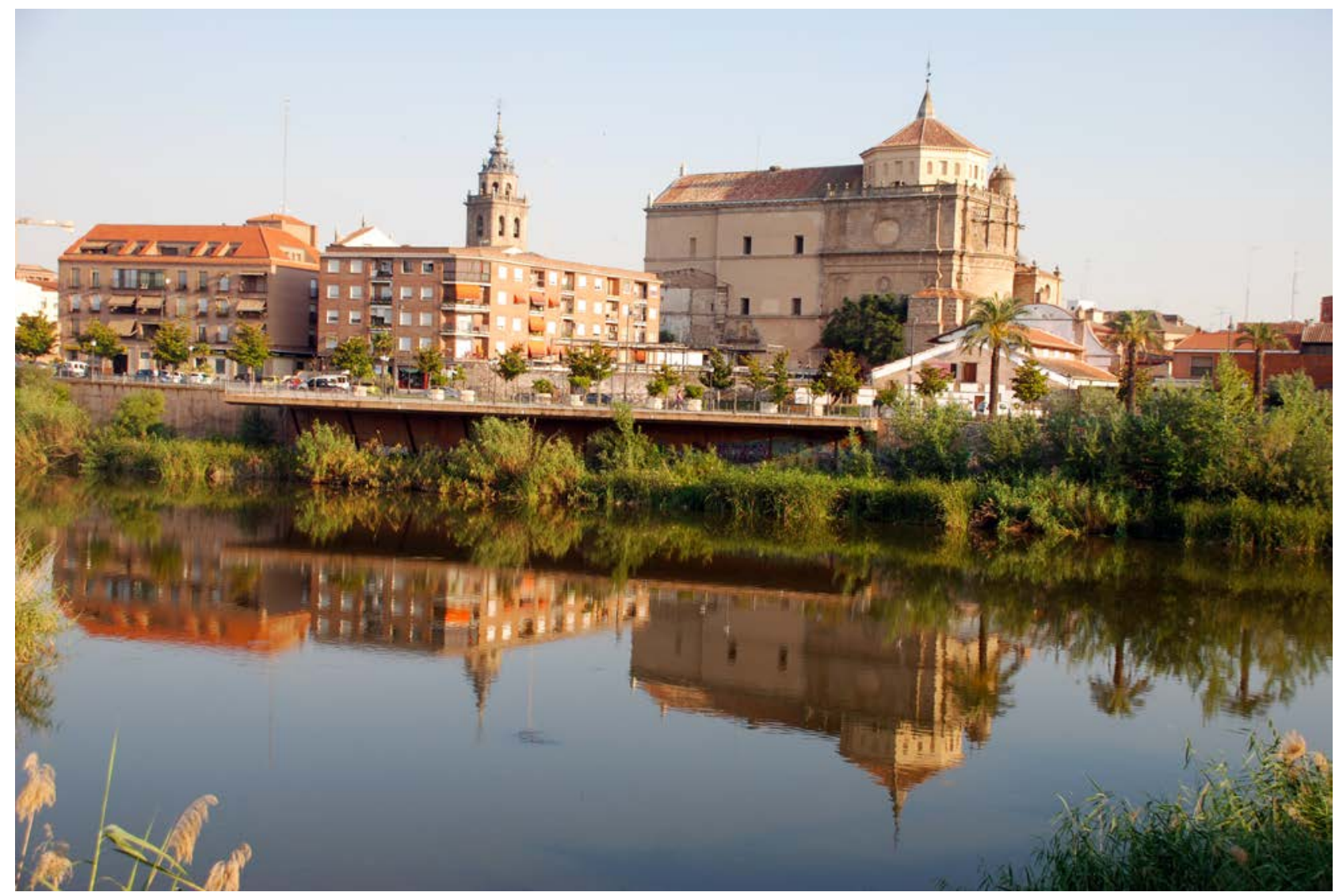

Fuente: De la Llave, 2018

Fig. 4 Vista de Talavera de la Reina y su entorno fluvial desde Puente Viejo

De forma paralela resulta indispensable optimizar la comunicación interdepartamental del consistorio. Al igual que la actualización de algunas normas y ordenanzas que puedan estar directa u indirectamente vinculadas al patrimonio cultural. En este sentido, servicios como urbanismo, patrimonio, medio ambiente, oficina técnica o contratación deben ir de la mano en muchos aspectos para lograr una gestión eficaz.

En relación con la gestión de las acciones que se lleven a cabo en el Conjunto Histórico, se plantean varias posibilidades como la creación de un consorcio monumental, una fundación, una empresa pública, un organismo autónomo local, asumir las labores con medios municipales propios o la externalización de servicios. Citados modelos conllevan a su vez ventajas y desventajas que deberán ser abordadas en su debido momento.

Otra cuestión de notable relevancia es el uso y aplicación de cerámica artística en el paisaje urbano. Constituye una seña de identidad de la ciudad y está declarada como Bien Cultural Inmaterial a nivel regional (DOCM, 16 de octubre de 2015, pp. 27272-27676) e inscrita por la UNESCO (2019) en la Lista Representativa del Patrimonio Cultural Inmaterial (PCI), durante el XIV Comité Intergubernamental para la Salvaguardia del PCI celebrado entre los días 9 y 14 de 
diciembre de 2019 (14.COM 10.B.23 - No. 01462). Al respecto, resulta imprescindible establecer criterios transversales sobre estética, localización, temática..., a fin de no "falsear o escenificar" el paisaje original del Conjunto Histórico.

Por su parte, es conveniente reseñar que la actual declaración no supone un marco cerrado. Nos referimos a la posibilidad de modificar el ámbito de la declaración, acometiendo la integración de nuevos espacios recogidos en el ámbito de protección, como es el caso de restos arqueológicos conservados en sótanos. Al respecto es preciso estudiar fórmulas destinadas a la adquisición de este tipo de espacios mediante permutas, cesiones o licencias extraordinarias que afecten al inmueble que se pretenda construir (alturas, superficie de ocupación, etc.). En el mismo sentido debemos indicar la necesidad de incluir el entorno del rio Tajo. La integración dentro de la declaración del conjunto del entorno ribereño requiere un estudio y justificación pormenorizada. El paisaje fluvial del Tajo a su paso por Talavera de la Reina puede considerarse bajo los términos de "paisaje cultural" (Fig. 4), pues cuenta con aspectos naturales y culturales (tangibles e intangibles) que deben ser tenidos en cuenta. En este sentido, resulta complicado entender que el complejo hidroeléctrico de la Isla de los Molinos, el cual fue de los primeros en producir energía a nivel regional y provincial, esté fuera de la declaración pero sí en el ámbito de protección (De la Llave y García, 2018).

En definitiva, como se puede comprobar, son numerosos los retos y tareas pendientes que tiene por delante el consistorio la ciudad para abordar la difícil, pero no imposible, tarea de gestionar su Conjunto Histórico.

\section{Referencias}

Acuerdo de 13/10/2015, por el que se declara Bien de Interés Cultural la Cerámica de Talavera de la Reina (Toledo), con la categoría de Bien Inmaterial. [2015/12402]. Diario Oicial de Castilla-La Mancha, 203, 16 de octubre de 2015, 27672-27676. Recuperado de https://docm.castillalamancha.es/portaldocm/detalleDocumento.do?idDisposicion=1444843344872860623

Acuerdo de 19/02/2019, por el que se declaran Bien de Interés Cultural los Jardines del Prado en el municipio de Talavera de la Reina (Toledo), con la categoría de Jardín Histórico. [2019/1869]. Diario Oficial de Castilla-La Mancha, 42, 28 de febrero de 2019, 6279-6288. Recuperado de

https://docm.castillalamancha.es/portaldocm/detalleDocumento.do?idDisposicion=1550683235027530088

Acuerdo de 19/02/2019, por el que se declara Bien de Interés Cultural el Conjunto Histórico de Talavera de la Reina (Toledo), con la categoría de Conjunto Histórico. [2019/1870]. Diario Oficial de Castilla-La Mancha, 42, 28 de febrero de 2019, 6289-6317. Recuperado de https://docm.castillalamancha.es/portaldocm/detalleDocumento.do?idDisposicion=1550682339890530078

Alguacil, M. C., Maquedano, B., y Molina, N. (2017). Informe técnico sobre la solicitud de declaración de Talavera de la Reina (Toledo) como Bien de Interés Cultural con la categoría de Conjunto Histórico. Toledo: Viceconsejería de Cultura de Castilla-La Mancha.

De la Llave, S., y García, J. C. (2018). Aproximación a la producción eléctrica en Talavera de la Reina (Toledo): El complejo hidroeléctrico de los Molinos de Arriba. En C. Pacheco (Coord.), Actas del Congreso El Agua en la provincia de Toledo: historia, usos y retos para el futuro (pp. 243-262). Talavera de la Reina: Colectivo de Investigación Histórica Arrabal.

Ezquiaga, J. M. (2008). Plan de Ordenación Municipal Talavera de la Reina (Toledo). Talavera de la Reina: Ayuntamiento de Talavera de la Reina.

Ley 4/2013, de 16 de mayo, de Patrimonio Cultural de Castilla-La Mancha. [2013/6396]. Diario Oficial de Castilla-La Mancha, 100, 24 de mayo de 2013, 14189-14221. Recuperado de https://docm.castillalamancha.es/portaldocm/detalleDocumento.do?idDisposicion=1369043760043810037

Resolución de 15/11/2018, por la que se inicia expediente para la declaración de la Villa de Talavera de la Reina (Toledo) como Bien de Interés Cultural, con la categoría de Conjunto Histórico. [2018/13587]. Diario Oficial de Castilla-La Mancha, 226, 20 de noviembre de 2018, 30851-30880. Recuperado de https://docm.castillalamancha.es/portaldocm/detalleDocumento.do?idDisposicion=1542359777766060216

Rodríguez-Gimeno, S. (2018). Talavera de la Reina, Conjunto Histórico. Talavera de la Reina: Ayuntamiento de Talavera de la Reina.

UNESCO (2019). Convention for the safeguarding of the intangible cultural heritage. Intergovernmental Committee for the Safeguarding of the Intangible Cultural Heritage. Fourteenth session. Bogotá. 9 to 14 December 2019. Recuperado de https://ich.unesco.org/en/decisions/14.COM/10.B.23 